Anaesthesist 2009 - 58:5-6

DOI 10.1007/s00101-008-1497-5

Online publiziert: 21. Januar 2009

๑) Springer Medizin Verlag 2009
S.C. Azad

Klinik für Anaesthesiologie, Klinikum Großhadern, Ludwig-Maximilians-Universität München

\section{Opioidinduzierte Immunsuppression als klinisch relevantes Problem?}

\section{Im Zweifel für den Angeklagten!}

Im Jahr 1806 gelang dem jungen Apotheker Friedrich Wilhelm Sertürner in Einbeck die Extraktion des schlaffördernden Alkaloids Morphin aus dem Mohnsaft. Durch Acetylierung des Morphins entwickelte C. Wright 1874 das halbsynthetische Derivat Diacetylmorphin, das als Heroin bekannt ist. Seit dieser Zeit wurde kontinuierlich eine Reihe vollsynthetischer Substanzen dieser Stoffgruppe entwickelt, die uns heute zur Therapie unserer Patienten zur Verfügung steht. Stammen diese direkt aus dem Opium, wie Morphin oder Kodein, werden sie als Opiate bezeichnet, während die vollsynthetischen Stoffe Opioide genannt werden. Im Rahmen der Allgemein- und Regionalanästhesie werden vorwiegend hochpotente, eher kurz wirksame Opioide wie Fentanyl oder Sufentanil eingesetzt. Zur Therapie akuter und chronischer Schmerzen gibt es eine Vielzahl hoch- und niederpotenter Opioide, die meisten davon inzwischen in retardierter und unretardierter Form. Opiate und Opioide entfalten ihre Wirkung über eine Bindung an spezifische Opioidrezeptoren, die unter physiologischen Bedingungen durch entsprechende endogene Liganden besetzt werden.

Das endogene Opioidsystem besteht im Wesentlichen aus drei Subtypen von Opioidrezeptoren, den $\mu$-, $\delta$ - und $\kappa$-Rezeptoren, sowie den endogenen Opioidpeptiden $\beta$-Endorphin, Enzephalin und Dynorphin. Opioidrezeptoren sind metabotrope Rezeptoren, die an inhibitorische $\mathrm{G}_{\mathrm{i} / \mathrm{o}}$-Proteine gekoppelt sind. Wer- den sie im Zentralnervensystem (ZNS) aktiviert, führt dies vorwiegend über eine Aktivierung von Kaliumkanälen und eine Inhibierung von spannungsabhängigen Kalziumkanälen zu einer Hyperpolarisation der Neurone und zu einer Depolarisationshemmung. Die Folge ist insgesamt eine Verminderung der Erregbarkeit der Neurone und somit der Weiterleitung der neuronalen Aktivität. Opioidrezeptoren sind im Körper von Mensch und Tier an sehr vielen Stellen zu finden. Sie sind im ZNS und im peripheren Nervensystem (PNS), aber auch in vielen peripheren Organen wie beispielsweise dem Magen-Darm-Trakt exprimiert. Nach heutigem Kenntnisstand ist das endogene Opioidsystem eines der bedeutsamsten schmerzhemmenden Systeme des Körpers. Sinn dieses Systems ist es unter anderem, afferente Reize so zu modulieren, dass sie nicht ungehindert in das ZNS weitergeleitet werden, und somit zu vermeiden, dass es zu einer Übererregung und einer gesteigerten Schmerzempfindung auf schmerzhafte sowie nichtschmerzhafte Reize kommt.

Da Opioidrezeptoren im Körper so weit verbreitet sind, entfalten endogene und exogene Opioide auch sehr viele unterschiedliche Wirkungen: erwünschte, wie Analgesie, und unerwünschte, wie Übelkeit, Atemdepression und Obstipation. Besonders wichtig für die Anästhesie und die Schmerztherapie ist deren analgetische Wirkung. Diese wird über eine Aktivierung von Opioidrezeptoren im Thalamus, im periaquäduktalen Grau und im limbischen System (supraspinale Analgesie) sowie im Hinterhorn des Rückenmarks (spinale Analgesie) hervorgerufen. Daneben gelang der Arbeitsgruppe um Christoph Stein bereits in den 9oer Jahren die Aufklärung von wesentlichen Mechanismen der peripheren Opioidanalgesie. Sie fand heraus, dass sich auch auf peripheren sensorischen Neuronen Opioidrezeptoren befinden, die unter Entzündungsbedingungen hochreguliert werden. Dies geschieht über eine Steigerung der Bildung der Rezeptorproteine im Zellkern der Neurone und deren axonalen Transport in die Peripherie. Dort führt deren Aktivierung über endogene oder exogene Opioidrezeptorliganden zu einer Hemmung der Schmerzentstehung. Interessanterweise spielen dabei Immunzellen eine wichtige Rolle. Sie wandern in das entzündete Gewebe ein und synthetisieren Opioidpeptide, die sie dann unter bestimmten Bedingungen, wie einem Anstieg von „corticotropin-releasing factor" unter Stress, in unmittelbarer Nähe der Opioidrezeptoren freisetzen. Ein enger Zusammenhang zwischen dem endogenen Opioidsystem und dem Immunsystem ist daher schon lange bekannt. Die Frage, die sich stellt und die die Autoren Rittner und Brack im folgenden Leitartikel beantworten, ist nur, ob sich durch diese Interaktion im Gegenzug auch eine opioidinduzierte Immunsupression ergibt, die möglicherweise ein klinisch relevantes Problem werden könnte. 
Wie in der Arbeit von Rittner und Brack beschrieben, zeigen etliche Studien, die in Zellkulturen oder im Tiermodell durchgeführt wurden, dass Opioide eine immunmodulierende und -suppressive Wirkung haben und dadurch das Infektionsrisiko erhöhen können. Doch wie sieht dies in klinischen Studien aus? Hier befassen sich die Autoren intensiv mit der Relevanz dieses Problems bei Patienten mit retroviralen Infektionen (i.e. „human immunodeficiency virus“, HIV), Sepsis und Pneumonie und im Rahmen der Therapie postoperativer sowie chronischer Schmerzen. Sie kommen zu dem Schluss, dass die Datenlage diesbezüglich insgesamt unzureichend und widersprüchlich ist. Beispielsweise gibt es einen gewissen Zusammenhang zwischen Opioidgabe und erhöhter Infektionsrate bei Patienten mit Verbrennungen; gleichzeitig führte aber die Gabe von Morphin in einer placebokontrollierten Studie bei Frühgeborenen nicht zu einer Erhöhung der Sepsisinzidenz. Es wird sehr schnell klar, dass das Problem, wie so oft, in der Unzulänglichkeit vieler klinischer Studien liegt. Meist sind die Patientenzahlen für eine eindeutige Aussage zu klein, und oft drängt sich eine Fragestellung auf, die schon aus ethischen Gründen in klinischen Studien nicht wirklich fundiert untersucht werden kann. Letzteres macht es neben der Tatsache, dass im Menschen fast jeder physische und psychische Vorgang multifaktoriell ist, nahezu unmöglich, eine gezielte, einzelne Interaktion $\mathrm{zu}$ untersuchen.

So ziehen die Autoren letztlich das Fazit, „dass ein Zusammenhang zwischen der Opioidgabe und einem erhöhten Infektionsrisiko bislang weder überzeugend belegt, noch sicher ausgeschlossen werden kann."

Eines ist jedoch sicher: Das endogene Opioidsystem hat eine wichtige physiologische Funktion. Dadurch, dass es im ganzen Körper aktiv ist (im ZNS, im PNS und in peripheren Organen), führt dessen Aktivierung auch zu vielfältigen Reaktionen, unter anderem zu einer Interaktion mit dem Immunsystem. Folgendes ist aber auch sicher: Trotz aller Nebenwirkungen von Opioiden und entsprechenden Kontraindikationen sind die Erfindung von Morphin durch Sertürner und die Entwicklung der weiteren Opioide für die Menschheit ein Segen. Denn sowohl im Rahmen der Anästhesie als auch der postoperativen Schmerztherapie, der Therapie von chronischen tumorbedingten und vielen nichttumorbedingten Schmerzen, der Intensiv- und Palliativmedizin ist deren Einsatz im Grunde unverzichtbar.

Die Frage ist nur, ob es Patientengruppen gibt, bei denen eine Opioidgabe das Infektionsrisiko deutlich erhöhen und somit von Nachteil sein kann, z. B. bei immunsupprimierten Patienten oder Intensivpatienten. Diese Frage bleibt weiterhin interessant und wichtig, bei dem derzeitigen Wissensstand jedoch noch offen. So gilt bis zum Beweis des Gegenteils: „Im Zweifel für den Angeklagten!“

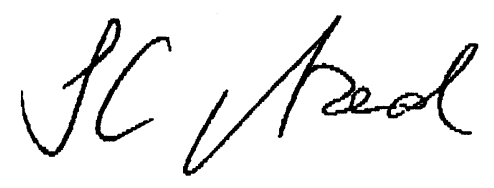

\section{Korrespondenzadresse \\ PD Dr. S.C. Azad}

Klinik für Anaesthesiologie, Klinikum Großhadern, Ludwig-Maximilians-Universität München Marchioninistr. 15, 81377 München

Shahnaz.Azad@med.uni-muenchen.de

Interessenkonflikt. Die korrespondierende Autorin gibt an, dass kein Interessenkonflikt besteht.

\section{Literatur}

1. De Leon-Casasola O (2008) Current developments in opioid therapy for management of cancer pain. Clin J Pain 24:S3-S7

2. Dy SM, Asch SM, Naeim A et al (2008) Evidencebased standards for cancer pain management. J Clin Oncol 26:3879-3885

3. Jage J, Jurna I (2001) Opioidanalgetika. In: Zenz M, Jurna I (Hrsg) Lehrbuch der Schmerztherapie, 2. Aufl. Wissenschaftliche Verlagsgesellschaft, Stuttgart, S 255-280

4. Jage J, Laufenberg-Feldmann R, Heid F (2008) Medikamente zur postoperativen Schmerztherapie: Bewährtes und Neues. Anaesthesist 57:491-498

5. Schäfer M (1999) Peripheral opioid analgesia: from experimental to clinical studies. Curr Opin Anesthesiol 12:603-607

6. Stein C (1995) Mechanisms of disease: the control of pain in peripheral tissue by opioids. N Engl J Med 332:1685-1690

\section{Das Ambulante Operieren vor dem Aus?}

Ab 2009 tritt die neue Honorarsystematik für alle Vertragsärzte in Kraft. Dadurch kann es im Bereich des Ambulanten Operierens in einigen Bundesländern zu Einnahmeausfällen von bis zu 23\% kommen, so Berechnungen der Kommission der niedergelassenen Anästhesisten im Berufsverband Deutscher Anästhesisten. In anderen Bundesländern würde das bisherige $\mathrm{Ni}$ veau allenfalls gehalten. Vertragsärzte und Krankenhäuser sind von diesen Kürzungen gleichermaßen betroffen. Die Kommission befürchtet, dass die neue Honorarsystematik zu einer Gefährdung der wohnortnahen Versorgung bei Ambulanten Operationen sowie einem Verlust von mehr als 10.000 Arbeitsplätzen führen wird. Daher seien Krankenkassen und Kassenärztliche Vereinigung nun gefordert unverzüglich Maßnahmen zu treffen, um Ambulante Operationen und Anästhesien angemessen zu vergüten. Zumal die Reform in den Medien als Honorarsteigerung von $\sim 10 \%$ präsentiert wurde.

Durch Ambulante Operationen konnten bisher ohne Qualitätsverlust schon erhebliche Einsparungen erzielt werden. Dieser Bereich könnte bei angemessener Honorierung noch deutlich wachsen und somit weitere Einsparungen von mehr als zwei Milliarden Euro erreichen.

Quelle: Berufsverband Deutscher Anästhesisten, www.bda.de 